\title{
Patient and partner empowerment programme for idiopathic pulmonary fibrosis
}

To the Editor:

Idiopathic pulmonary fibrosis (IPF) is a progressive, deadly disease with a devastating impact on patients' and their partners' quality of life (QoL) [1]. Many studies have shown the need for support groups, better information resources and disease education in IPF [2-6]. Although these needs have been identified, few studies currently exist concerning interventions that can fulfil them and possibly improve QoL for patients and their partners [7]. In our study, we determined the effect of a short multidisciplinary empowerment programme on the QoL for patients with IPF and their partners.

In 2014 and 2015, consecutive IPF outpatients and their partners at the Erasmus MC, in Rotterdam, were asked to participate in a Patient and Partner Empowerment Programme for IPF, called PPEPP. "Partner" was broadly defined as spouse, partner, family member or close friend. PPEPP consisted of three afternoon meetings, divided over three consecutive weeks and focuses on coping with IPF. A psychologist who is experienced in group therapy leads PPEPP. A pulmonologist, specialised interstitial lung disease nurse, oxygen supplier, social worker and physiotherapists also contribute to the sessions. The protocol and content were designed by the participating disciplines. Moreover, two patients with IPF, a former physiotherapist and a vicar, gave their input on the protocol and content of the programme. A comprehensive description of the programme and the contribution of each discipline can be found in the supplementary material.

Patients and partners were included in three blocks: two intervention groups and one control group. Patients were included if they had been diagnosed with IPF according to the guidelines of 2011 [8], had a life expectancy of $\geqslant 1$ year, had a lung function with a forced vital capacity (FVC) $\geqslant 45 \%$ of predicted, and had a diffusion capacity for carbon monoxide ( $D \mathrm{LCO}) \geqslant 25 \%$ of predicted. Participants were asked to fill in questionnaires at baseline, after 3 weeks and after 3 months. All participants filled in the Hospital Anxiety and Depression Scale (HADS), Perceived Stress Scale (PSS) and a knowledge quiz about IPF [9]. Furthermore, patients completed the King's Brief Interstitial Lung Disease health status questionnaire (K-BILD), the Euroqol5D5L (EQ5D5L) and the Medical Research Council (MRC) dyspnoea scale, while their partners completed the Carer Quality of Life instrument (CarerQoL) [10-12]. We also asked participants to complete an evaluation form after PPEPP. The Wilcoxon signed rank test was used to compare baseline scores with follow-up scores, because the data were not normally distributed. Medical ethics committee approval was obtained, and all participants gave written informed consent.

In total, 46 participants were included, 15 couples in the intervention group (eight couples in the first, seven couples in the second) and eight in the control group. In the intervention group, two couples were excluded. One couple could not participate because of clinical worsening of IPF, and the other couple did not complete baseline questionnaires and missed the first meeting because of an influenza infection. In the control group, one patient died of heart failure after 3 weeks, thereby excluding this couple from analysis.

In the intervention group, most patients were men (10,77\%) and most partners were women (10, 77\%); patients had a median age of 63 (range 54-74) years and partners of 64 (47-74) years; the median FVC in patients was $80 \%(50-100 \%)$ of predicted and DLCO was $46 \%(25-60 \%)$ of predicted. In the control group, all patients were men and all partners were women; patients had a median age of 76 (63-82) years and

@ERSpublications

PPEPP, a short multidisciplinary empowerment programme, improves quality of life for IPF patients and their partners http://ow.ly/zAbb3096waC

Cite this article as: van Manen MJG, van 't Spijker A, Tak NC, et al. Patient and partner empowerment programme for idiopathic pulmonary fibrosis. Eur Respir J 2017; 49: 1601596 [https://doi.org/10.1183/ 13993003.01596-2016]. 
partners of 74 (22-84) years; the median FVC in patients was $78 \%(53-96 \%)$ of predicted and DLCO was $48 \%(30-82 \%)$ of predicted.

Both groups were matched for disease severity defined by pulmonary function. However, baseline questionnaire scores differed except for HADS depression, PSS and CarerQoL (table 1). Questionnaire scores significantly improved after 3 weeks of PPEPP (table 1) in the intervention group only.

After a 3-month follow-up, no significant changes in questionnaire scores compared with baseline were found in the intervention group. In the control group, QoL measures were lower at 3 months than at baseline: K-BILD total median of 72 (range 45-82) versus 46 (31-86), p=0.03; K-BILD psychological domain 65 (38-73) versus 47 (15-72), $\mathrm{p}=0.03$; EQ5D5L 0.9 (0.7-1.0) versus 0.8 (0.3-0.8), $\mathrm{p}=0.03$; and HADS total $6(0-22)$ versus 7 (1-28), $\mathrm{p}=0.04$. After 3 months, FVC deteriorated $\geqslant 5 \%$ in one patient of the control group and in none of the intervention groups.

All participants considered PPEPP useful and would recommend it to others, and 25 participants (96\%) found PPEPP fulfilled their expectations. The following quotes illustrate the participants' experiences with PPEPP: "informative, useful and supportive", "pleasant to share experience with and learn from peers", "comforting to know that you're not alone in your struggles".

This study showed that a short multidisciplinary empowerment programme improved QoL for patients with IPF and their partners. To our knowledge, ours is the first study in which the effect of a support programme, co-developed with patients and multidisciplinary experts, demonstrated a positive effect on the wellbeing of patients and their partners.

Currently, it is well acknowledged that there is a need for better information and support for patients with IPF and their partners. Many hospitals organise general IPF information meetings and support groups; however, studies on effective ways of supporting and educating patients and partners are scarce. A previous study by LINDELL et al. [7] on the effect of a 6-week programme on disease management and symptom reduction showed, strikingly, a decline in patients' QoL and increased anxiety levels. Nevertheless, in partners, stress levels decreased, and interviews showed that participants found attending the programme helpful. It is useful to realise that information can be distressing to patients and should be tailored carefully. In our study, perceived stress scores showed no differences in stress levels. The co-development by two patient experts may have been a factor in helping to tailor our programme more effectively.

PPEPP consists of small groups, which stimulate personal interaction, and can be more patient tailored than general information meetings, which are often made up of large groups. In our opinion, the experience of the psychologist with group therapy was crucial in stimulating discussion and promoting balanced participation for all. The extensive experience with group counselling in the field of oncology could also prove useful. With IPF bearing similarities in both prognosis and treatment options with oncology, we think we could learn from their experience [13-15].

TABLE 1 Wilcoxon signed rank test intervention and control group; baseline versus week 3

\begin{tabular}{|c|c|c|c|c|c|c|c|}
\hline & \multirow[t]{2}{*}{ Questionnaires } & \multicolumn{3}{|c|}{ Intervention group ( $n=26)$} & \multicolumn{3}{|c|}{ Control group ( $n=14)$} \\
\hline & & Baseline & Week 3 & p-value & Baseline & Week 3 & $p$-value \\
\hline$\wedge$ & K-BILD total & $43(28-69)$ & $46(32-81)$ & 0.06 & $72(45-82)$ & $61(34-79)$ & 0.17 \\
\hline$\wedge$ & K-BILD psych & $49(22-72)$ & $52(30-78)$ & 0.03 & $65(38-73)$ & $63(17-80)$ & 0.87 \\
\hline$\wedge$ & EQ5D5L & $0.6(0.4-1.0)$ & $0.7(0.6-09)$ & 0.07 & $0.9(0.7-1.0)$ & $0.9(0.6-1.0)$ & 0.40 \\
\hline$\vee$ & MRC & $3(2-5)$ & $3(2-5)$ & 0.32 & $2(0-3)$ & $2(2-4)$ & 0.18 \\
\hline v & HADS total & $11(3-26)$ & $9(0-27)$ & 0.04 & $6(0-22)$ & $7(1-23)$ & 0.31 \\
\hline 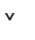 & HADS anxiety & $6(1-15)$ & $5(0-14)$ & 0.06 & $4(0-13)$ & $5(0-12)$ & 0.14 \\
\hline$\vee$ & HADS depression & $5(0-15)$ & $4(0-13)$ & 0.04 & $3(0-10)$ & $2(1-12)$ & 0.52 \\
\hline$\checkmark$ & PSS total & $20(5-40)$ & $21(5-33)$ & 0.94 & $23(11-33)$ & $23(4-34)$ & 0.48 \\
\hline v & CarerQoL & $3(0-10)$ & $3(0-6)$ & 0.25 & $2(0-3)$ & $2(0-3)$ & 0.71 \\
\hline$\wedge$ & CarerQoL VAS & $7(5-9)$ & $7(5-10)$ & 0.86 & $8(7-9)$ & $8(6-9)$ & 0.45 \\
\hline ^ & IPFquiz & $6(3-9)$ & $7(3-9)$ & 0.27 & $4(2-6)$ & $4(1-9)$ & 0.86 \\
\hline
\end{tabular}

Data are presented as median (range). K-BILD: King's Brief Interstitial Lung Disease health status questionnaire; psych: psychological domain; EQ5D5L: Euroqol5D5L; MRC: Medical Research Council dyspnoea scale; HADS: Hospital Anxiety and Depression Scale; PSS: Perceived Stress Scale; CarerQoL: Carer Quality of Life instrument; VAS: visual analogue scale; IPF: idiopathic pulmonary fibrosis. ^: a higher score indicates better quality of life/knowledge on disease; ${ }^{\vee}$ : a higher score indicates worse breathlessness/ anxiety/depression/stress/quality of life. Bold indicates statistically significant $p$-values. 
PPEPP improved short-term QoL but showed no effect long-term. Research into more chronic support is needed as different stages of disease often mandate adaptation of coping strategies for patients and partners [1].

This study has some limitations. First, it consists of small groups of patients from a single centre, and though the results are encouraging, further studies are needed. Another limitation is the difference in baseline QoL scores between the intervention and control groups. We lack a good explanation for this, as no significant differences existed in disease severity defined by pulmonary function (FVC and DLCO). Including patients in blocks (for practical reasons) instead of randomising them may have influenced results. Still, participants were not allowed to choose between groups, and the control group was offered the opportunity to attend a future PPEPP. For future studies, it would be worth exploring the effect of matching participants based on their QoL scores instead of pulmonary function.

In conclusion, PPEPP, a concise multidisciplinary empowerment programme, improves short-term quality of life for patients with IPF and their partners. Patients and partners were very satisfied with PPEPP. More research, however, is needed to develop structural support programmes for patients and partners throughout the disease course.

Mirjam J.G. van Manen ${ }^{1}$, Adriaan van 't Spijker ${ }^{2}$, Nelleke C. Tak ${ }^{1}$, Carla T. Baars ${ }^{3}$, Sandra M. Jongenotter ${ }^{3}$, Liesbeth R. van Roon ${ }^{4}$, Jitske Kraan ${ }^{1}$, Henk C. Hoogsteden ${ }^{1}$ and Marlies S. Wijsenbeek ${ }^{1}$

${ }^{1}$ Dept of Respiratory Medicine, Erasmus Medical Center, University Hospital Rotterdam, Rotterdam, The Netherlands. ${ }^{2}$ Dept of Psychiatry, Section Medical Psychology and Psychotherapy, Erasmus Medical Center, University Hospital Rotterdam, Rotterdam, The Netherlands. ${ }^{3}$ Unit Physiotherapy, Erasmus Medical Center, University Hospital Rotterdam, Rotterdam, The Netherlands. ${ }^{4}$ Unit Psychosocial Care, Erasmus Medical Center, University Hospital Rotterdam, Rotterdam, The Netherlands.

Correspondence: M.S. Wijsenbeek, Erasmus MC, University Hospital Rotterdam, Dept of Pulmonary Disease, 's-Gravendijkwal 230, Rotterdam, 3015 CE, The Netherlands E-mail: m.wijsenbeek-lourens@erasmusmc.nl

Received: Aug 112016 | Accepted after revision: Jan 152017

This article has supplementary material available from erj.ersjournals.com

Conflict of interest: Disclosures can be found alongside this article at erj.ersjournals.com

Acknowledgements: We respectfully acknowledge the valuable input given by $\mathrm{Mr}$ van der Steen and $\mathrm{Mr}$ van Poppel, whose personal and professional experiences have provided indispensable guidance for design of the PPEPP programme.

\section{References}

1 Overgaard D, Kaldan G, Marsaa K, et al. The lived experience with idiopathic pulmonary fibrosis: a qualitative study. Eur Respir J 2016; 47: 1472-1480.

2 Belkin A, Albright K, Swigris JJ. A qualitative study of informal caregivers' perspectives on the effects of idiopathic pulmonary fibrosis. BMJ Open Respir Res 2013: 1; e000007.

3 Thickett DR, Kendall C, Spencer LG, et al. Improving care for patients with idiopathic pulmonary fibrosis (IPF) in the UK: a round table discussion. Thorax 2014; 69: 1136-1140.

4 Bonella F, Wijsenbeek M, Molina-Molina M, et al. European IPF Patient Charter: unmet needs and a call to action for healthcare policymakers. Eur Respir J 2016; 47: 597-606.

5 Russell AM, Sprangers MA, Wibberley S, et al. The need for patient-centred clinical research in idiopathic pulmonary fibrosis. BMC Med 2015; 13: 240.

6 Fisher JH, O'Connor D, Flexman AM, et al. Accuracy and reliability of Internet resources for information on idiopathic pulmonary fibrosis. Am J Respir Crit Care Med 2016; 194: 218-225.

7 Lindell KO, Olshansky E, Song MK, et al. Impact of a disease-management program on symptom burden and health-related quality of life in patients with idiopathic pulmonary fibrosis and their care partners. Heart Lung 2010; 39: 304-313.

8 Raghu G, Collard HR, Egan JJ, et al. An official ATS/ERS/JRS/ALAT statement: idiopathic pulmonary fibrosis: evidence-based guidelines for diagnosis and management. Am J Respir Crit Care Med 2011; 183: 788-824.

9 Cohen S, Kamarck T, Mermelstein R. A global measure of perceived stress. J Health Soc Behav 1983; 24: 385-396.

10 Patel AS, Siegert RJ, Brignall K, et al. The development and validation of the King's Brief Interstitial Lung Disease (K-BILD) health status questionnaire. Thorax 2012; 67: 804-810.

11 Devlin NJ, Krabbe PF. The development of new research methods for the valuation of EQ-5D-5L. Eur J Health Econ 2013; 14: Suppl. 1, S1-S3.

12 Hoefman RJ, van Exel J, Brouwer WB. Measuring the impact of caregiving on informal carers: a construct validation study of the CarerQol instrument. Health Qual Life Outcomes 2013; 11: 173.

13 Dockham B, Schafenacker A, Yoon $\mathrm{H}$, et al. Implementation of a psychoeducational program for cancer survivors and family caregivers at a cancer support community affiliate: a pilot effectiveness study. Cancer Nurs 2016; 39: 169-180.

14 Risendal BC, Dwyer A, Seidel RW, et al. Meeting the challenge of cancer survivorship in public health: results from the evaluation of the chronic disease self-management program for cancer survivors. Front Public Health 2014; 2: 214 .

15 Vancheri C. Idiopathic pulmonary fibrosis and cancer: do they really look similar? BMC Med 2015; 13: 220. 